\title{
Various types of phase transitions in the AdS soliton background
}

\author{
Yan Peng \\ Department of Physics, Fudan University, 200433 Shanghai, China \\ Qiyuan Pan, Bin Wang \\ INPAC and Department of Physics, Shanghai Jiao Tong University, 200240 Shanghai, China
}

\begin{abstract}
We study the basic holographic insulator and superconductor phase transition in the AdS soliton background by generalizing the spontaneous breaking of a global U(1) symmetry to occur via Stückelberg mechanism. We construct the soliton solutions with backreaction and examine the effects of the backreaction on the condensation of the scalar hair in the generalized Stückelberg Lagrangian. We disclose rich physics in various phase transitions. In addition to the AdS soliton configuration, we also examine the property of the phase transition in the AdS black hole background.

PACS numbers: $11.25 . \mathrm{Tq}, 04.70 . \mathrm{Bw}, 74.20 .-\mathrm{z}$
\end{abstract}

\section{INTRODUCTION}

The AdS/CFT correspondence [1 3$]$ has provided a framework to describe the strongly coupled field theories in a weakly coupled gravitational system. In recent years, this correspondence has been applied to study the holographic model of superconductors, which is constructed by a gravitational theory of a Maxwell field coupled to a charged complex scalar field [4-6]. It has been shown that the bulk AdS black hole becomes unstable and scalar hair condenses below a critical temperature. In the boundary dual CFT, many properties shared by the superconductor have been exhibited. The instability of the bulk black hole corresponds to a second order phase transition from normal state to superconducting state which brings the spontaneous U(1) symmetry breaking. The gravity models with the property of the holographic superconductor have attracted considerable interest for their potential applications to the condensed matter physics, see for example [7]- [30].

Considering that the spontaneous breaking of a global U(1) symmetry in the basic holographic superconductor model occurs via the Stückelberg mechanism, recently it was disclosed that besides the second order phase transition, a fairly wide class of phase transitions can be allowed to happen [31]. The Stückelberg mechanism allows tuning the order of the phase transition which can accommodate the first order phase transition to occur, and for the second order phase transition it allows tuning the values of critical exponents [32]. An interesting 
extension was done in [33] by constructing general models for holographic superconductivity. Rich phenomena in the phase transition were also found for the holographic superconductors in Einstein-Gauss-Bonnet gravity where the Gauss-Bonnet coupling can play the role in determining the order of phase transition and critical exponents in the second-order phase transition [34].

Besides the bulk AdS black hole spacetime, recently a superconducting phase dual to an AdS soliton configuration was studied in [35]. The AdS soliton is a gravitational configuration which has lower energy than the AdS space in the Poincaré coordinates, but has the same boundary topology as the Ricci flat black hole and the AdS space in the Poincaré coordinates [36]. There is a first order phase transition called Hawking-Page phase transition between the Ricci flat AdS black hole and the AdS soliton [37]. The signature of this phase transition was shown up in the quasi-normal modes spectrum [38]. The Hawking-Page phase transition between Ricci flat black holes and deformed AdS soliton in the Gauss-Bonnet gravity was discussed in [39]. In [35], another phase transition was disclosed in the AdS soliton configuration. It was shown that if one adds a chemical potential $\mu$ to the AdS soliton, there is a second order phase transition at a critical value $\mu_{c}$, beyond which the charge scalar field turns on, even at zero temperature. This is an insulator/superconductor transition. The analysis in [35] was performed in the limit of the probe approximation, where the backreaction of the matter fields is not taken into account. Considering the backreaction of the matter fields on the soliton geometry, a new phase boundary in which a first order phase transition was observed for the same chemical potential when the backreaction is strong enough [40]. The various phase transitions of gravity duals of superfluid/fluid/insulator in $(2+1)$-dimensions have been investigated in [41].

In this work we will extend the discussion on the basic holographic insulator/superconductor transition in the AdS soliton background by generalizing the spontaneous breaking of a global U(1) symmetry to occur via Stückelberg mechanism. We will construct the soliton solutions with backreaction, examine the effects of the backreaction on the condensation of the scalar hair in the generalized Stückelberg Lagrangian and disclose rich physics in various phase transitions. In addition to the AdS soliton background, we will also examine the property of the phase transition in AdS black hole background in the generalized Stückelberg formalism.

\section{VARIOUS PHASE TRANSITIONS IN THE ADS SOLITON BACKGROUND}

\section{A. The bulk equations}

In this section, we study in detail the formation of the scalar hair in the 5-dimensional AdS soliton spacetime. The generalized action containing a U(1) gauge field and the scalar field coupled via a generalized St $\ddot{u}$ ckelberg 
Lagrangian reads [31, 32, 34]

$$
S=\int d^{5} x \sqrt{-g}\left[\left(R+\frac{12}{l^{2}}\right)+L_{m a t t e r}\right]
$$

where $l$ is the AdS radius which will be taken to be unity in the following discussion. $L_{\text {matter }}$ is the generalized Stückelberg Lagrangian

$$
L_{\text {matter }}=-\frac{1}{4} F^{\mu \nu} F_{\mu \nu}-(\partial \psi)^{2}-m^{2}|\psi|^{2}-\left(\psi^{2}+q^{2} c_{4} \psi^{4}\right)(\partial p-q A)^{2},
$$

where $\psi(r)$ and $A_{\mu}$ are the scalar and Maxwell fields coupled to gravity. $c_{4}$ is a model parameter. Setting $q A=\tilde{A}$ and considering the gauge symmetry $\tilde{A}_{\mu} \rightarrow \tilde{A}_{\mu}+\partial \Lambda$ and $p \rightarrow p+\Lambda$, we can fix the gauge $p=0$ by using the gauge freedom.

We are interested in including the backreaction so we choose the metric ansatz

$$
d s^{2}=r^{2}\left[e^{A(r)} B(r) d \eta^{2}+d x^{2}+d y^{2}-e^{C(r)} d t^{2}\right]+\frac{d r^{2}}{r^{2} B(r)} .
$$

We require that $B$ vanishes at some radius $r_{s}$, which corresponds to the tip of the soliton. As in the usual AdS soliton, smoothness at the tip requires that $\eta$ be periodic with the period

$$
\gamma=\frac{4 \pi e^{-A\left(r_{s}\right) / 2}}{r_{s}^{2} B^{\prime}\left(r_{s}\right)}
$$

Choosing the electromagnetic field and the scalar field as

$$
\psi=\psi(r), \quad A=\phi(r) d t
$$

we can obtain the equations of motion of matter fields

$$
\begin{gathered}
\psi^{\prime \prime}+\left(\frac{5}{r}+\frac{A^{\prime}}{2}+\frac{B^{\prime}}{B}+\frac{C^{\prime}}{2}\right) \psi^{\prime}+\frac{q^{2} \phi^{2} e^{-C}}{r^{4} B}\left(\psi+2 q^{2} c_{4} \psi^{3}\right)-\frac{m^{2}}{r^{2} B} \psi=0, \\
\phi^{\prime \prime}+\left(\frac{3}{r}+\frac{A^{\prime}}{2}+\frac{B^{\prime}}{B}-\frac{C^{\prime}}{2}\right) \phi^{\prime}+\frac{2 q^{2} \phi}{r^{2} B}\left(\psi^{2}+q^{2} c_{4} \psi^{4}\right)=0 .
\end{gathered}
$$

The nontrivial components, such as tt, rr, xx, yy, $\eta \eta$ components in Einstein equations are not all independent. From the xx-tt and $\eta \eta+t t-x x$ components, we have

$$
\begin{gathered}
C^{\prime \prime}+\frac{1}{2} C^{\prime 2}+\left(\frac{5}{r}+\frac{A^{\prime}}{2}+\frac{B^{\prime}}{B}\right) C^{\prime}-\left[\phi^{\prime 2}+\frac{2 q^{2} \phi^{2}}{r^{2} B}\left(\psi^{2}+q^{2} c_{4} \psi^{4}\right)\right] \frac{e^{-C}}{r^{2}}=0, \\
B^{\prime}\left(\frac{3}{r}-\frac{C^{\prime}}{2}\right)+B\left(\psi^{\prime 2}-\frac{1}{2} A^{\prime} C^{\prime}+\frac{e^{-C} \phi^{\prime 2}}{2 r^{2}}+\frac{12}{r^{2}}\right)+\frac{q^{2} \phi^{2} e^{-C}}{r^{4}}\left(\psi^{2}+q^{2} c_{4} \psi^{4}\right)+\frac{1}{r^{2}}\left(m^{2} \psi^{2}-12\right)=0 .
\end{gathered}
$$

Substracting the $\eta \eta$ component from the rr equation, we get

$$
A^{\prime}=\frac{2 r^{2} C^{\prime \prime}+r^{2} C^{2}+4 r C^{\prime}+4 r^{2} \psi^{\prime 2}-2 e^{-C} \phi^{2}}{r\left(6+r C^{\prime}\right)}
$$


We need to integrate these equations from the tip of the soliton out to the infinity. Since the equations are coupled and nonlinear, we have to count on the numerical calculation. At the tip $r_{s}$, we can do the expansion

$$
\begin{array}{r}
\psi(r)=a+b\left(r-r_{s}\right)+c\left(r-r_{s}\right)^{2}+\cdots, \\
\phi(r)=\alpha+\beta\left(r-r_{s}\right)+\gamma\left(r-r_{s}\right)^{2}+\cdots, \\
B(r)=u\left(r-r_{s}\right)+\cdots, \\
C(r)=v+w\left(r-r_{s}\right)+\cdots, \\
A(r)=A_{0}+A_{1}\left(r-r_{s}\right)+\cdots
\end{array}
$$

Putting these Taylor expansions into equations, we get four independent parameters: $a, A_{0}, \alpha, v$. At $r \rightarrow \infty$, after choosing $m^{2}=-\frac{15}{4}$ above the BF bound for $A d S_{5}: m_{B F}^{2}=-\frac{(D-1)^{2}}{4}=-4[42]$, the scalar and Maxwell equations have the form

$$
\psi=\frac{\psi_{-}}{r^{3 / 2}}+\frac{\psi_{+}}{r^{5 / 2}}+\cdots, \phi=\mu-\frac{\rho}{2 r^{2}}+\cdots
$$

and parameters $C(\infty)=0, A(\infty)=0, B(\infty)=1$. At our chosen value of $m^{2}$, both $\psi_{-}$or $\psi_{+}$are normalizable. We fix $\psi_{-}=0$ in our following discussion. For given values of $a$, we can obtain the required numerical solutions from the differential equations above.

Using the scaling symmetry

$$
r \rightarrow a r, \quad(t, x, y, \eta) \rightarrow(t, x, y, \eta) / a, \quad \phi \rightarrow a \phi
$$

we can set $r_{s}=1$. After getting the solutions, these symmetry can be used again to scale $\gamma=\pi$. Other quantities and operators are scaled as: $\gamma \rightarrow \frac{1}{a} \gamma, \mu \rightarrow \mathrm{a} \mu, \rho \rightarrow a^{3} \rho, \psi_{+}^{5 / 2} \rightarrow \mathrm{a} \psi_{+}^{5 / 2}$.

\section{B. Effects on the phase transition}

It was shown in [24, 35] that AdS soliton experiences a second order phase transition, the insulator/superconductor phase transition, around the critical chemical potential $\mu_{c}$. But these results were got in the probe limit which was justified for large $q$. Taking the backreaction into account, it was observed that when $q$ is reduced to $q \approx 1$, the second order phase transition will give way to the first order phase transition[40].

It is of interest to see how the generalized Stückelberg formalism plays the role in the phase transition in the AdS soliton background. When $c_{4}=0$, our result goes back to that disclosed in [40] that the first order phase transition appears only when $q \sim 1$ and for $q>1$ there is only the second order phase transition. For $q>1$, we need to turn on $c_{4}$ to accommodate the first order phase transition. For the chosen strength of the backreaction 
with $q>1$, we can find a threshold value of $c_{4}$. Above this threshold $c_{4}$, the condensate operator together with the charge density $\rho$ do not have a monotonic behavior, which indicates the appearance of the first order phase transition. When we increase $q$, we find that the threshold $\bar{c}_{4}$ becomes bigger. We show the numerical behaviors of the condensate and the charge density with the change of the chemical potential in Fig.1 and Fig.2.
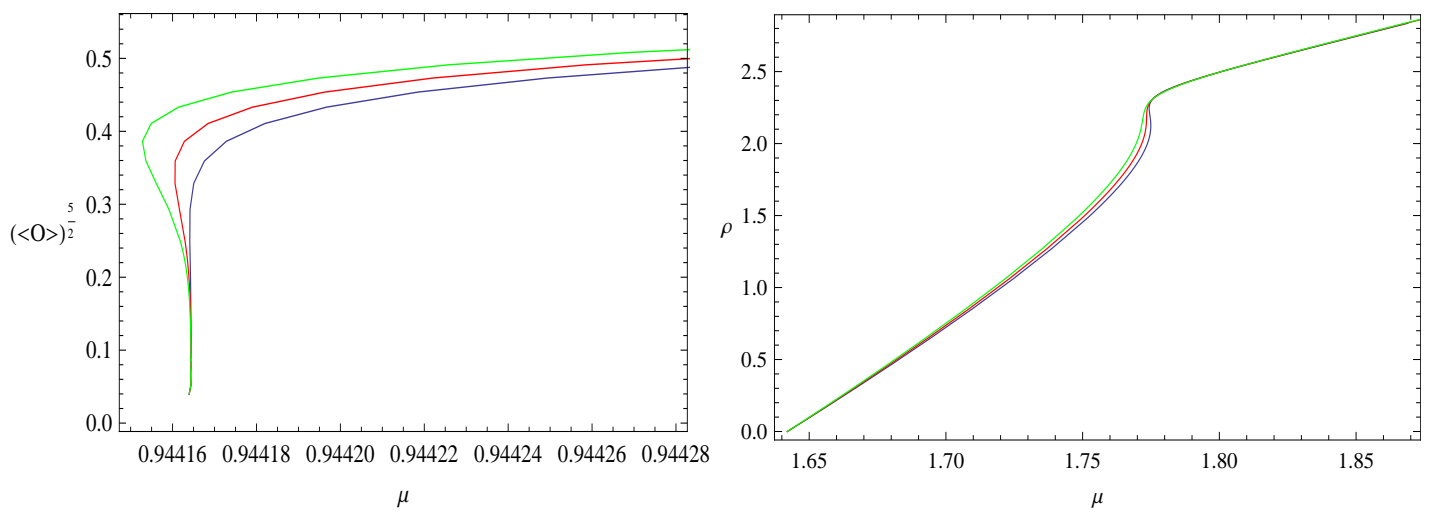

FIG. 1: (Color online) The left panel shows the value of the condensate $\langle O\rangle$ as a function of chemical potential $\mu$ when $q=2$ with $c_{4}=0.471$ (blue), $c_{4}=0.473$ (red), $c_{4}=0.475$ (green), respectively. The right panel shows the charge density $\rho$ as a function of chemical potential $\mu$ with $\mathrm{q}=1.15$. $c_{4}$ decreases from left to right, i.e., $c_{4}=0.11$ (green), $c_{4}=0.105$ (red) and $c_{4}=0.1$ (blue).

We observe the influence of the backreaction on the scalar condensation in the AdS soliton background. For choosing the same value of the scalar mass, qualitative features occur as we vary the backreaction of the matter field on the AdS soliton background. As we increase $q$, the condensation gap becomes smaller, which means that the scalar hair can be formed easier in the more weakly backreacted background. The gap is not sensitive to the model parameter $c_{4}$.

It is interesting to ask what is the upper bound on $\bar{c}_{4}$ when $q$ grows to infinity, namely when the backreaction disappears. In order to answer this question, we set $q \psi=\tilde{\psi}, q \phi=\tilde{\phi}, \frac{1}{q^{2}}=\lambda$, so that

$$
\begin{gathered}
\tilde{\psi}^{\prime \prime}+\left(\frac{5}{r}+\frac{A^{\prime}}{2}+\frac{B^{\prime}}{B}+\frac{C^{\prime}}{2}\right) \tilde{\psi}^{\prime}+\frac{\tilde{\phi}^{2} e^{-C}}{r^{4} B}\left(\tilde{\psi}+2 c_{4} \tilde{\psi}^{3}\right)-\frac{m^{2}}{r^{2} B} \tilde{\psi}=0 \\
\tilde{\phi}^{\prime \prime}+\left(\frac{3}{r}+\frac{A^{\prime}}{2}+\frac{B^{\prime}}{B}-\frac{C^{\prime}}{2}\right) \tilde{\phi}^{\prime}-\frac{2 \tilde{\phi}}{r^{2} B}\left(\tilde{\psi}^{2}+c_{4} \tilde{\psi}^{4}\right)=0 \\
C^{\prime \prime}+\frac{1}{2} C^{\prime 2}+\left(\frac{5}{r}+\frac{A^{\prime}}{2}+\frac{B^{\prime}}{B}\right) C^{\prime}-\lambda\left[\tilde{\phi}^{\prime 2}+\frac{2 \tilde{\phi}^{2}}{r^{2} B}\left(\tilde{\psi}^{2}+c_{4} \tilde{\psi}^{4}\right)\right] \frac{e^{-C}}{r^{2}}=0, \\
B^{\prime}\left(\frac{3}{r}-\frac{C^{\prime}}{2}\right)+B\left(\lambda \tilde{\psi}^{\prime 2}-\frac{1}{2} A^{\prime} C^{\prime}+\lambda \frac{e^{-C} \tilde{\phi}^{\prime 2}}{2 r^{2}}+\frac{12}{r^{2}}\right)+\lambda \frac{\tilde{\phi}^{2} e^{-C}}{r^{4}}\left(\tilde{\psi}^{2}+c_{4} \tilde{\psi}^{4}\right)+\frac{1}{r^{2}}\left(\lambda m^{2} \tilde{\psi}^{2}-12\right)=0, \\
A^{\prime}=\frac{2 r^{2} C^{\prime \prime}+r^{2} C^{\prime 2}+4 r C^{\prime}+4 r^{2} \lambda \tilde{\psi}^{\prime 2}-2 e^{-C} \lambda \tilde{\phi}^{\prime 2}}{r\left(6+r C^{\prime}\right)} .
\end{gathered}
$$


$q \rightarrow \infty$ corresponds to $\lambda \rightarrow 0$ which reduces all equations above to the probe limit. Solving the above equations numerically, we find $\bar{c}_{4}=0.57$ when $q \rightarrow \infty$, which means that even in the probe limit, when $c_{4}$ is above this threshold value, the first order phase transition can substitute the second order phase transition to happen in the AdS soliton background. This is qualitatively similar to the findings in the AdS black hole configurations 32, 34]. For clarity, in Table $\llbracket$ we list the values of the threshold $\bar{c}_{4}$ for different strength of the backreaction in the AdS soliton background.

TABLE I: The threshold value $\bar{c}_{4}$ which can accommodate the first order phase transition for different strength of the backreaction in the AdS soliton background.

\begin{tabular}{|c|c|c|c|c|c|c|}
\hline $\mathrm{q}$ & 1.0 & 1.5 & 1.7 & 2.0 & 5.0 & $\infty$ \\
\hline $\bar{c}_{4}$ & 0.000 & 0.230 & 0.450 & 0.473 & 0.555 & 0.570 \\
\hline
\end{tabular}

In the above discussion we fixed the strength of the backreaction and examined the threshold of $c_{4}$ in the generalized Stückelberg formalism to change the order of the phase transition from the second to the first. We have also examined the effects of the change of the strength of the backreaction on the order of the phase transition for the chosen $c_{4}$. We find that for the chosen nonzero $c_{4}$, the condensate exhibits non-monotonic behavior for smaller $q$. This means that the first order of the phase transition can appear easily when the backreaction is stronger for the fixed $c_{4}$.

\section{VARIOUS PHASE TRANSITIONS IN THE ADS BLACK HOLE BACKGROUND}

\section{A. The bulk equations}

Now we turn our discussion to the AdS black hole background. To include the backreaction, we consider the metric ansatz

$$
d s^{2}=-g(r) e^{-\chi(r)} d t^{2}+\frac{d r^{2}}{g(r)}+r^{2}\left(d x^{2}+d y^{2}+d z^{2}\right)
$$

The event horizon $r_{H}$ satisfies $g\left(r_{H}\right)=0$ and the Hawking temperature is expressed as $T=\frac{g^{\prime}\left(r_{H}\right) e^{-\chi\left(r_{H}\right) / 2}}{4 \pi}$. Using the symmetry

$$
r \rightarrow a r,(t, x, y, z) \rightarrow(t, x, y, z) / a, g \rightarrow a^{2} g, \phi \rightarrow a \phi
$$

we can set the horizon $r_{H}=1$.

Assuming the matter fields in the form of (5), we get the equations of motion

$$
\begin{gathered}
\psi^{\prime \prime}+\left(\frac{3}{r}-\frac{\chi^{\prime}}{2}+\frac{g^{\prime}}{g}\right) \psi^{\prime}+\frac{q^{2} \phi^{2} e^{\chi}}{g^{2}}\left(\psi+2 q^{2} c_{4} \psi^{3}\right)-\frac{m^{2}}{g} \psi=0 \\
\phi^{\prime \prime}+\left(\frac{3}{r}+\frac{\chi^{\prime}}{2}\right) \phi^{\prime}-\frac{2 q^{2} \phi}{g}\left(\psi^{2}+q^{2} c_{4} \psi^{4}\right)=0
\end{gathered}
$$




$$
\begin{gathered}
\chi^{\prime}+\frac{2 r \psi^{\prime}}{3}+\frac{2 r q^{2} \phi^{2} e^{\chi}}{3 g^{2}}\left(\psi^{2}+q^{2} c_{4} \psi^{4}\right)=0, \\
g^{\prime}+\left(\frac{2}{r}-\frac{\chi^{\prime}}{2}\right) g+\frac{r e^{\chi} \phi^{\prime 2}}{6}+\frac{m^{2} r \psi^{2}}{3}-4 r=0 .
\end{gathered}
$$

For the Maxwell field which is regular at the horizon, we can choose $\phi\left(r_{H}\right)=0$. The solution around the horizon can be expanded as

$$
\begin{aligned}
& \psi(r)=a^{\prime}+b^{\prime}\left(r-r_{H}\right)+c^{\prime}\left(r-r_{H}\right)^{2}+\cdots, \\
& \phi(r)=\alpha^{\prime}\left(r-r_{H}\right)+\beta^{\prime}\left(r-r_{H}\right)^{2}+\cdots, \\
& g(r)=u^{\prime}\left(r-r_{H}\right)+\cdots, \\
& \chi(r)=v^{\prime}+w^{\prime}\left(r-r_{H}\right)+\cdots .
\end{aligned}
$$

Substituting them into the equations of motion, we find that $a^{\prime}, u^{\prime}, v^{\prime}$ are independent. For the mass $m^{2}=-\frac{15}{4}$, the scalar and Maxwell fields have the same asymptotic behavior just as shown in Eq. (16). For simplicity, we will choose $a^{\prime}$ as the parameter to adjust the solution to satisfy $\psi_{-}=0$ and $\chi(\infty)=0$. We can integrate these equations numerically from the horizon out to infinity.

\section{B. Effects on the phase transition}

After solving the differential equations numerically, we can examine the phase transition in the AdS black hole background. When $c_{4}=0$, the phase transition always remains second order in the AdS black hole background even when $q<1$, which supports the observation in [40]. The second order phase transition will give way to the first order only when we turn on $c_{4}$ in the generalized Stückelberg formalism. In the probe limit when $q \rightarrow \infty$, we obtain $\bar{c}_{4}=2.28$. Below this $\bar{c}_{4}$, the phase transition is of the second order, while the first order phase transition is allowed when $c_{4}>2.28$. When the backreaction of the matter fields to the AdS black hole background is not strong enough, the behavior of the condensation due to the change of $c_{4}$ was shown in Fig.4. The condensate operator appears a non-monotonic behavior which indicates the appearance of the first order phase transition. We find that when $q$ is not small enough, with the decrease of the value of $q$, the threshold value of $c_{4}$ to allow the first order phase transition to happen becomes smaller.

However the threshold $\bar{c}_{4}$ will not decrease monotonically with the decrease of $q$ (the increase of the backreaction). When the backreaction is strong enough for some certain small value of $q$, with the decrease of $q$, the threshold $\bar{c}_{4}$ will increase to allow the first order phase transition to happen. For different chosen values of $q$, the thresholds $\bar{c}_{4}$ to accommodate the first order phase transition are listed in table II. 

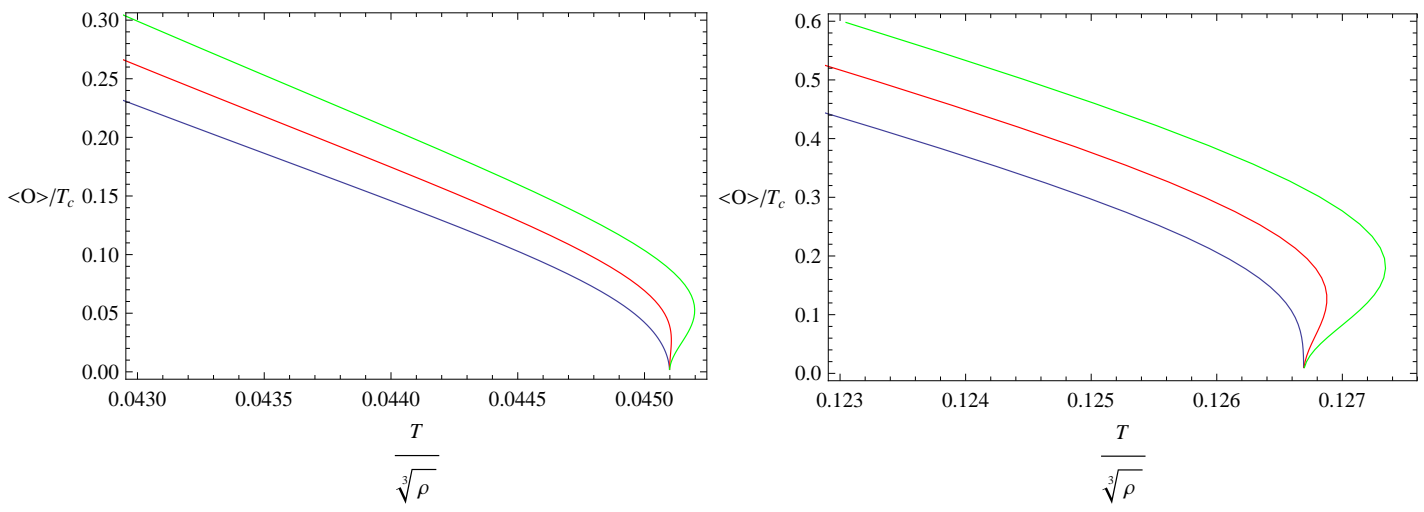

FIG. 2: (Color online) The value of the condensate $\langle O\rangle$ as a function of the temperature $T$. The left column represents the case when $q=1$ and the three lines from left to right correspond to the increase of $c_{4}$, i.e., $c_{4}=0.74$ (blue), $c_{4}=0.75$ (red), $c_{4}=0.76$ (green), the right one is for $q=1.5$ with $c_{4}$ changing $\left(c_{4}=0.4\right.$ (blue), $c_{4}=0.425($ red $), c_{4}=0.45($ green) $)$.

TABLE II: The threshold value $\bar{c}_{4}$ which can accommodate the first order phase transition for different strength of the backreaction in the AdS black hole background.

\begin{tabular}{|c|c|c|c|c|c|c|}
\hline $\mathrm{q}$ & 1.0 & 1.5 & 2.0 & 5.0 & 10.0 & $\infty$ \\
\hline $\bar{c}_{4}$ & 0.75 & 0.43 & 0.53 & 1.55 & 2.05 & 2.28 \\
\hline
\end{tabular}

From the equations of motion (25)-(28), we see that the $c_{4}$ is always related to $q^{4}$. This implies that in the generalized Stückelberg formalism in order to show the effect of $c_{4}$ when $q$ approaches zero, $c_{4}$ must tend to infinity. To check this, more careful numerical calculations are needed. However, when $q<1$, our numerical calculation becomes not efficient and more time consuming with the decrease of $q$.

\section{CONCLUSIONS AND DISCUSSIONS}

We have studied the possibility to allow various phase transitions to happen in the AdS soliton configuration. In the probe limit, it was argued that only second order phase transition can happen in the AdS soliton background $[24,35]$. Considering the backreaction of the matter field to the background spacetime, it was found that when the effect of the backreaction is strong enough, for example with $q \sim 1$, the second order phase transition will give way to the first order phase transition between the insulator and superconductor in the AdS soliton configuration [41]. It is of interest to study the phase transition in the generalized Stückelberg formalism, which has been applied in the AdS black hole backgrounds [31,32,34]. Applying the generalized Stückelberg formalism to the AdS soliton configuration, we find that there is always a threshold which can change the phase transition from the second order to the first order. When the backreaction of the matter field becomes weaker, the threshold in the generalized Stückelberg formalism to accommodate the first order phase transition is bigger. But there is an upper limit of this threshold, above this upper limit the first order phase transition can happen even in the probe limit in the AdS soliton configuration. In the AdS soliton case, the transition of the second order phase 
transition into the first order is natural as there is already Hawking-page transition which is the first order.

We have extended our discussion to the AdS black hole background. It was argued in [40] that in the AdS black hole spacetime, no matter how strong the backreaction is considered, even with $q<1$, there only exists the second order phase transition between the conductor and superconductor. In the generalized St $\ddot{u}$ ckelberg formalism, we observed rich physics in the phase transition in the AdS black hole configuration. When the backreaction is weak, there is a threshold and above which the first order phase transition can happen. The threshold value becomes smaller when the backreaction becomes bigger. However when the backreaction is very strong, the first order phase transition will be hard to happen in the AdS black hole configuration. For the very strong backreaction, the relation between the threshold and the backreaction is different from that for the weak backreaction case, the threshold increases when the backreaction becomes much stronger. Counting on the big effect of the $\Psi^{4}$ term in the generalized St $\ddot{u}$ ckelberg formalism, we found that the first order phase transition can be accommodated in the strong backreaction situation, for example with $q<1$.

When the backreaction is very strong with $q$ much smaller than unity, we argued that the threshold value to accommodate the first order phase transition will experience a fast grow. This argument still needs further accurate numerical check. Our present numerical code becomes inefficient and very time consuming when the backreaction $q<1$. More precise calculations are called for.

In [43] it was argued that transforming the Abelian Higgs model in three dimensions (i.e. the Ginzburg-Landau theory) to a disorder field theory, there is a critical point where the second order phase transition changes to the first order, which happens for a specific range of the Ginzburg-Landau parameter. Our observation of the change of the order of the phase transition in the AdS black hole background gives a very similar example in the gravity side. This shows a further example of the correspondence between the properties in AdS spacetime and the condensed matter physics.

\section{Acknowledgments}

This work was partially supported by NNSF of China.

[1] J.M. Maldacena, Adv. Theor. Math. Phys. 2, 231 (1998).

[2] S.S. Gubser, I.R. Klebanov, and A.M. Polyakov, Phys. Lett. B 428, 105 (1998).

[3] E. Witten, Adv. Theor. Math. Phys. 2, 253 (1998).

[4] S.A. Hartnoll, Class. Quant. Grav. 26, 224002 (2009).

[5] C.P. Herzog, J. Phys. A 42, 343001 (2009).

[6] G.T. Horowitz, arXiv:1002.1722 [hep-th].

[7] G.T. Horowitz and M.M. Roberts, Phys. Rev. D 78, 126008 (2008).

[8] E. Nakano, W.Y. Wen, Phys. Rev. D 78, 046004 (2008).

[9] I. Amado, M. Kaminski, and K. Landsteiner, J. High Energy Phys. 0905, 021 (2009). 
[10] G. Koutsoumbas, E. Papantonopoulos, and G. Siopsis, J. High Energy Phys. 0907, 026 (2009).

[11] O.C. Umeh, J. High Energy Phys. 0908, 062 (2009).

[12] H.B. Zeng, Z.Y. Fan, and Z.Z. Ren, Phys. Rev. D 80, 066001 (2009).

[13] J. Sonner, Phys. Rev. D 80, 084031 (2009).

[14] S.S. Gubser, C.P. Herzog, S.S. Pufu, and T. Tesileanu, Phys. Rev. Lett. 103, 141601 (2009).

[15] J.P. Gauntlett, J. Sonner, and T. Wiseman, Phys. Rev. Lett. 103, 151601 (2009).

[16] R.G. Cai and H.Q. Zhang, Phys. Rev. D 81, 066003 (2010).

[17] J.L. Jing and S.B. Chen, Phys. Lett. B 686, 68 (2010).

[18] C.P. Herzog, Phys. Rev. D 81, 126009 (2010).

[19] S.B. Chen, L.C. Wang, C.K. Ding, and J.L. Jing, Nucl. Phys. B 836, 222 (2010).

[20] R.A. Konoplya and A. Zhidenko, Phys. Lett. B 686, 199 (2010).

[21] G. Siopsis and J. Therrien, J. High Energy Phys. 1005, 013 (2010).

[22] K. Maeda, M. Natsuume, and T. Okamura, Phys. Rev. D 79, 126004 (2009).

[23] R. Gregory, S. Kanno, and J. Soda, J. High Energy Phys. 0910, 010 (2009).

[24] Q.Y. Pan, B. Wang, E. Papantonopoulos, J. Oliveira, and A.B. Pavan, Phys. Rev. D 81, 106007 (2010).

[25] X.H. Ge, B. Wang, S.F. Wu, and G.H. Yang, J. High Energy Phys. 1008, 108 (2010).

[26] X. He, B. Wang, R.G. Cai, and C.Y. Lin, Phys. Lett. B 688, 230 (2010).

[27] R.G. Cai, Z.X. Nie, B. Wang, and H.Q. Zhang, arXiv:1005.1233 [gr-qc].

[28] Y. Brihaye and B. Hartmann, Phys. Rev. D 81, 126008 (2010).

[29] S.S. Gubser, Phys. Rev. D 78, 065034 (2008).

[30] S.A. Hartnoll, C.P. Herzog, and G.T. Horowitz, Phys. Rev. Lett. 101, 031601 (2008).

[31] S. Franco, A.M. Garcia-Garcia, and D. Rodriguez-Gomez, J. High Energy Phys. 1004, 092 (2010).

[32] S. Franco, A.M. Garcia-Garcia, and D. Rodriguez-Gomez, Phys. Rev. D 81, 041901(R) (2010).

[33] F. Aprile and J.G. Russo, Phys. Rev. D 81, 026009 (2010).

[34] Q.Y. Pan and B. Wang, Phys. Lett. B 693, 159 (2010).

[35] T. Nishioka, S. Ryu, and T. Takayanagi, J. High Energy Phys. 1003, 131 (2010).

[36] G.T. Horowitz and R.C. Myers, Phys. Rev. D 59, 026005 (1998).

[37] S. Surya, K. Schleich, and D.M. Witt, Phys. Rev. Lett. 86, 5231 (2001).

[38] J. Shen, B. Wang, R. K. Su, C.Y. Lin, and R.G. Cai, J. High Energy Phys. 0707, 037 (2007).

[39] R.G. Cai, S.P. Kim, and B. Wang, Phys. Rev. D 76, 024011 (2007).

[40] G.T. Horowitz and B. Way, J. High Energy Phys. 1011, 011 (2010).

[41] Y. Brihaye and B. Hartmann, arXiv:1101.5708 [hep-th].

[42] P. Breitenlohner and D.Z. Freedman, Phys. Lett. B 115, 197 (1982).

[43] H. Kleinert, Lett. Nuovo Cimento, 35, (1982) 405. 